\title{
LINC00460 Enhances Bladder Carcinoma Cell Proliferation and Migration by Modulating miR-612/FOXK1 Axis
}

\author{
Jianwei Li Sihuai Huang Yanmei Zhang Weifeng Zhuo Baocheng Tong \\ Fangzhen Cai \\ Department of Urology, The Second Affiliated Hospital of Fujian Medical University, Quanzhou, China
}

\section{Keywords}

LINC00460 · miR-612 · Forkhead Box K1 · Bladder carcinoma ·

Progression

\begin{abstract}
Introduction: LincRNA (long intergenic noncoding RNA) has been indicated as a mediator in tumorigenesis of bladder carcinoma. This study was performed to evaluate the role of LINC00460 in bladder carcinoma progression. Methods: Expression levels of LINC00460 in bladder carcinoma tissues and cell lines were analyzed via qRT-PCR. MTT, EdU (5-ethynyl2 '-deoxyuridine) staining, and colony formation assays were utilized to evaluate cell viability and proliferation. The wound healing assay was performed to evaluate bladder cancer cell migration, and the transwell assay was used to evaluate cell invasion. The microRNA (miRNA) target of LINC00460 and the corresponding target gene were validated via the dual luciferase activity assay. The tumorigenic function of LINC00460 was determined via establishment of a xenotransplanted tumor model. Results: LINC00460 was elevated in bladder carcinoma tissues and cell lines. Elevated LINC00460 was associated with shorter overall survival of bladder carcinoma patients. Overexpression of LINC00460 promoted cell viability, proliferation, invasion, and migration, while silencing of LINC00460 indicated the opposite ef-
\end{abstract}

karger@karger.com www.karger.com/pha

Karger $\frac{1}{\%}$

GOPEN ACCESS
(C) 2020 The Author(s)

Published by S. Karger AG, Basel

This is an Open Access article licensed under the Creative Commons Attribution-NonCommercial-4.0 International License (CC BY-NC) (http://www.karger.com/Services/OpenAccessLicense), applicable to the online version of the article only. Usage and distribution for commercial purposes requires written permission. fect on bladder carcinoma progression. LINC00460 could directly bind to miR-612 and inhibit miR-612 expression. Moreover, LINC00460 expression was negatively correlated with miR-612 in patients with bladder carcinoma. FOXK1 (Forkhead Box K1) was identified as the target of miR-612 and upregulated in patients with bladder carcinoma. Overexpression of FOXK1 attenuated interference of LINC00460-inhibited bladder carcinoma progression. Knockdown of LINC00460 suppressed in vivo bladder carcinoma growth. Conclusions: LINC00460 promoted bladder carcinoma progression via sponging miR-612 to facilitate FOXK1 expression, suggesting that LINC00460 might have the potential of being explored as a therapeutic target for treatment of bladder carcinoma.

(C) 2020 The Author(s)

Published by S. Karger AG, Basel

\section{Introduction}

Bladder carcinoma is one of the most common malignancies in the urinary tract [1]. Its incidence has been increasing year by year, with an average growth rate of $4.6 \%$ over the past 10 years [2]. Due to high recurrence rate and infiltrating ability, the 5-year survival rate of patients with advanced bladder carcinoma is only $6 \%$ [3]. Moreover, bladder carcinoma is difficult to diagnose, ow- 
ing to its different clinical manifestations and histopathological types [4]. Therefore, it is particularly important to explore effective biomarkers for bladder carcinoma and investigate the underlying mechanism contributing to bladder carcinoma progression.

Long noncoding RNAs (lncRNAs) are involved in gene transcription regulation, RNA processing, chromatin modification, cell life activities, and other biological functions [5], especially in cancer [6]. A large number of abnormally expressed lncRNAs were identified in bladder carcinoma by gene microarray analysis [7]. LncRNA UCA1 [8], linc-UBC1 [9], and other lncRNAs are elevated in bladder carcinoma, while MEG3 [10] and GAS5 [11] are downregulated. In addition, these $\ln \mathrm{RNAs}$ play a role in regulating bladder carcinoma progression.

LINC00460, an lncRNA transcribed from chromosome 13, was upregulated in esophageal squamous cell carcinoma [12], lung cancer [13], and nasopharyngeal carcinoma [14]. Moreover, LINC00460 promoted cancer cell proliferation, epithelial-mesenchymal transition, and invasion. Data from The Cancer Genome Atlas (TCGA) database showed that LINC00460 was also upregulated in bladder cancer [15], and LINC00460 promoted progression of bladder urothelial carcinoma [16]. However, the specific mechanism remains unclear.

LncRNAs could modify chromatin segments or act as microRNAs sponges to regulate the stability of targeting mRNAs [17]. LINC00460 has been shown to regulate miR-485-5p-mediated Raf1 to participate in papillary thyroid cancer progression [18]. miR-939-5p-mediated LIM domain kinase 2 was also implicated in LINC00460regulated colorectal cancer cell metastasis [19]. LINC00460 acted as miR-485-5p sponges to upregulate P21 (RAC1) activated kinase 1 during hepatocellular carcinoma development [20]. However, the microRNAs involved in LINC00460-mediated bladder carcinoma progression remains largely unknown.

FOX family functions as transcription factors to participate in various physiological processes, including cell survival [21] and cell progression [22]. Dysregulated FOX transcription factors were largely found in bladder carcinoma and identified as prognostic markers [23]. FOX could also promote bladder carcinoma progression [24]. FOXK1 was reported to be involved in miR-365-3p-mediated breast cancer [25], miR-195-5p-mediated lung cancer [26], miR-646-meidated gastric cancer [27], circMAN2B2/miR-1275-mediated lung cancer [28], and LINC01503/miR-4492-mediated colorectal cancer [29]. However, the role of FOXK1 in bladder carcinoma has not been reported.
Table 1. Primer sequences

\begin{tabular}{ll}
\hline ID & Sequence $\left(5^{\prime}-3^{\prime}\right)$ \\
\hline GAPDH F & ACCACAGTCCATGCCATCAC \\
GAPDH R & TCCACCACCCTGTTGCTGTA \\
LINC00460 F & GAAAGCTGCAACATGCTCCC \\
LINC00460 R & TCCATGTCGTCCCAGTTGGT \\
miR-612 F & GCAGGGCTTCTGAGCTCCTTAA \\
miR-612 R & CAAATTCGTGAAGCGTTCCATAT \\
FOXK1 F & ACACGTCTGGAGGAGACAGC \\
FOXK1 R & GAGAGGTTGTGCCGGATAGA \\
U6 F & CTCGCTTCGGCAGCACA \\
U6 R & AACGCTTCACGAATTTGCGT \\
\end{tabular}

The expression of LINC00460 in bladder carcinoma was first evaluated. Thereafter, the effects of LINC00460 on bladder carcinoma progression were determined. Furthermore, the downstream binding target was also explored, which would facilitate for the development of novel therapeutics to bladder carcinoma.

\section{Materials and Methods}

\section{Tumor Tissues Collection}

The research was approved by the Medical Ethics Committee of The Second Affiliated Hospital of Fujian Medical University, and all the patients signed written informed consent. Forty-three paired human bladder carcinoma and adjacent noncancer tissues were collected from patients via surgical resection at The Second Affiliated Hospital of Fujian Medical University. Patients were diagnosed via pathology examination.

\section{Cell Culture}

SV-HUC-1 (simian virus 40-imortalized human uroepithelial cells), as well as bladder carcinoma cell lines (T-24, 5637, SW780, RT-112), were cultured in the DMEM medium (Lonza, Basel, Switzerland) supplemented with $10 \%$ fetal bovine serum (FBS; Gibco, Waltham, MA, USA) at $37^{\circ} \mathrm{C}$ in an incubator with $5 \% \mathrm{CO}_{2}$.

\section{Cell Transfection}

For the overexpression of LINC00460 and FOXK1, sequences were constructed into pcDNA3.1 (Invitrogen, Carlsbad, CA, USA). For the knockdown of LINC00460, shRNAs (1\#: 5' -GCTAAGACCTAATAGCCAATA- $3^{\prime}$ and 2\#: $5^{\prime}$-ACCTTGGTCAAACGTTTAACC- $3^{\prime}$ ), as well as the negative control (sh-NC, 5'-GTTCTCCGAACGTGTACGT-3'), were constructed into pLKO.1 RiboBio (Guangzhou, China). HEK-293T cells were cotransfected with psPAX2 and pMD2.G with pLKO-shLINC00460 2\# (sh1LINC00460) or pLKO-shNC (Scramble). Forty-eight hours later, lentiviruses with the released lentiviral vectors were harvested. T-24 cells were infected with the lentiviruses using $8 \mathrm{mg} / \mathrm{mL}$ polybrene. Stable T-24 cell lines with shLINC00460 or shNC were
80

Pharmacology 2021;106:79-90 DOI: $10.1159 / 000509255$
Li/Huang/Zhang/Zhuo/Tong/Cai 


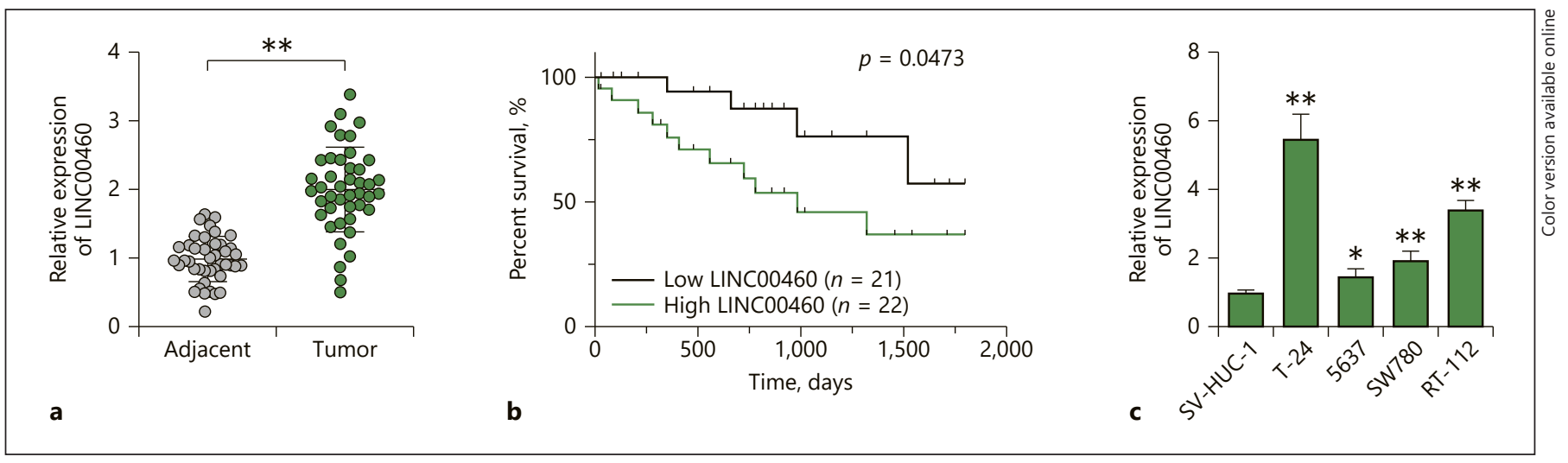

Fig. 1. LINC00460 was elevated in bladder carcinoma tissues and cell lines. a LINC00460 was upregulated in bladder carcinoma tissues compared to adjacent noncancer tissues, as detected by qRTPCR. ** represents tumor versus adjacent tissues, $p<0.01$. b High LINC00460 expression in bladder carcinoma patients showed

obtained with a treatment of $5 \mu \mathrm{g} / \mathrm{mL}$ puromycin for 1 week. MiR612 mimics (miR-612) or negative control (NC) and miR-612 inhibitor (miR-612 inh) or NC inh were obtained from GenePharma (Suzhou, China). 5637 or T-24 cells were then transfected with them via Lipofectamine 2000 (Invitrogen).

\section{Cell Proliferation Assay}

5637 or T24 cells $\left(2 \times 10^{3} /\right.$ well $)$ with different treatment were seeded in 96-well plates for $24 \mathrm{~h}$. For 4 consecutive days, cells were incubated with $0.1 \mathrm{mg} / \mathrm{mL}$ MTT (Roche Applied Science, Mannheim, Germany) at $37^{\circ} \mathrm{C}$ for $4 \mathrm{~h}$ and then lysed in dimethyl sulfoxide for $10 \mathrm{~min}$. Absorbance at $490 \mathrm{~nm}$ was measured with Microplate Autoreader (Bio-Rad, Hercules, CA, USA). For colony formation experiments, 5637 or T24 cells $\left(1 \times 10^{3} /\right.$ well $)$ were seeded into 6-well plates and the medium was replaced every 3 days. Fourteen days later, the colonies were fixed in methanol and stained with $0.1 \%$ crystal violet and photographed under a light microscope (Olympus, Tokyo, Japan). For 5-ethynyl-2'deoxyuridine (EdU) staining, $200 \mu \mathrm{L} 2 \times 10^{4} / \mathrm{mL} 5637$ or T2 4 cells were incubated with $50-\mu \mathrm{M}$ EdU for $8 \mathrm{~h}$ and then fixed in $70 \%$ alcohol for $15 \mathrm{~min}$. After permeabilization with Triton X-100 for 20 min, the cells were incubated with Apollo staining reaction liquid (Ribobio, Guangzhou, China). Immunostainings were then visualized using a fluorescent microscope (Olympus Inverted Microscope IX71, Tokyo, Japan) with DAPI (4',6-diamidino-2-phenylindole) staining.

\section{Wound Healing}

Gaps in seeded 5637 or T24 cells were generated via a plastic pipette tip. After removing the debris or the detached cells, the cells were cultured in DMEM for another $24 \mathrm{~h}$ before calculation of wound width by Wound Healing ACAS Image Analysis Software (Ibidi, Martinsried, Germany).

Transwell Assay

5637 or T24 cells were first seeded into the upper wells of chambers (BD Biosciences, Bedford, MA, USA) with a Matrigel-coated poor OS compared to low levels of LINC00460. c LINC00460 was upregulated in human bladder carcinoma cell lines (T-24, 5637, SW780, and RT-112) compared to SV-HUIC-1, as detected by qRT-PCR. *, ** represent bladder carcinoma cell lines versus SVHUIC- $1, p<0.05, p<0.01$.

membrane (BD Biosciences) in a 200- $\mu$ L FBS-free DMEM medium. The lower wells of the chambers were filled with $400-\mu \mathrm{L}$ DMEM medium containing 10\% FBS. Eight hours later, the medium of the upper wells and the filters were removed. Twenty-four hours later, the invasive cells were fixed with $100 \%$ methanol and then stained with $0.1 \%$ crystal violet for $1 \mathrm{~h}$. The stained cells were imaged under a microscope (Olympus).

\section{Dual Luciferase Reporter Assay}

Sequences of wildtype or mutant LINC00460 or 3'-UTR of FOXK1 were subcloned into the pmirGLO luciferase reporter vector (Promega, Madison, WI, USA). 5637 or T24 cells were first seeded and then cotransfected miR-612 or NC and miR-612 inh or NC inh with pmirGLO-wt-LINC00460, pmirGLO-mutLINC00460, pmirGLO-wt-FOXK1, or pmirGLO-mut-FOXK1. Two days after transfection, the luciferase activities were determined via Lucifer Reporter Assay System (Promega) and normalized to Renilla luciferase activity.

\section{$q R T-P C R$}

RNAs were isolated with Trizol (Invitrogen), and miRNAs were extracted via an miRcute miRNA isolation kit (Tiangen, Beijing, China). RNAs were then reverse-transcribed into cDNAs, and qRT-PCR was conducted with SYBR Green Master (Roche, Mannheim, Germany). GAPDH and U6 were used as endogenous controls. The primer sequences are shown in Table 1.

\section{Western Blot}

Thirty-microgram proteins were separated by SDS-PAGE and then electro-transferred onto PVDF membranes. After blocking with $5 \%$ BSA, the membranes were incubated with primary antibodies overnight: anti-FOXK1 (1:1,500; Abcam, Cambridge, MA, USA), anti-PCNA (1:2,000; Abcam), anti-E-cadherin (1:2,500; Abcam), and anti- $\beta$-actin (1:3,000; Abcam) at $4^{\circ} \mathrm{C}$. Following incubation with HRP-labeled secondary antibody (1:5,000; Abcam), the immunoreactivities were detected by enhanced chemiluminescence (KeyGen, Nanjin, China). 


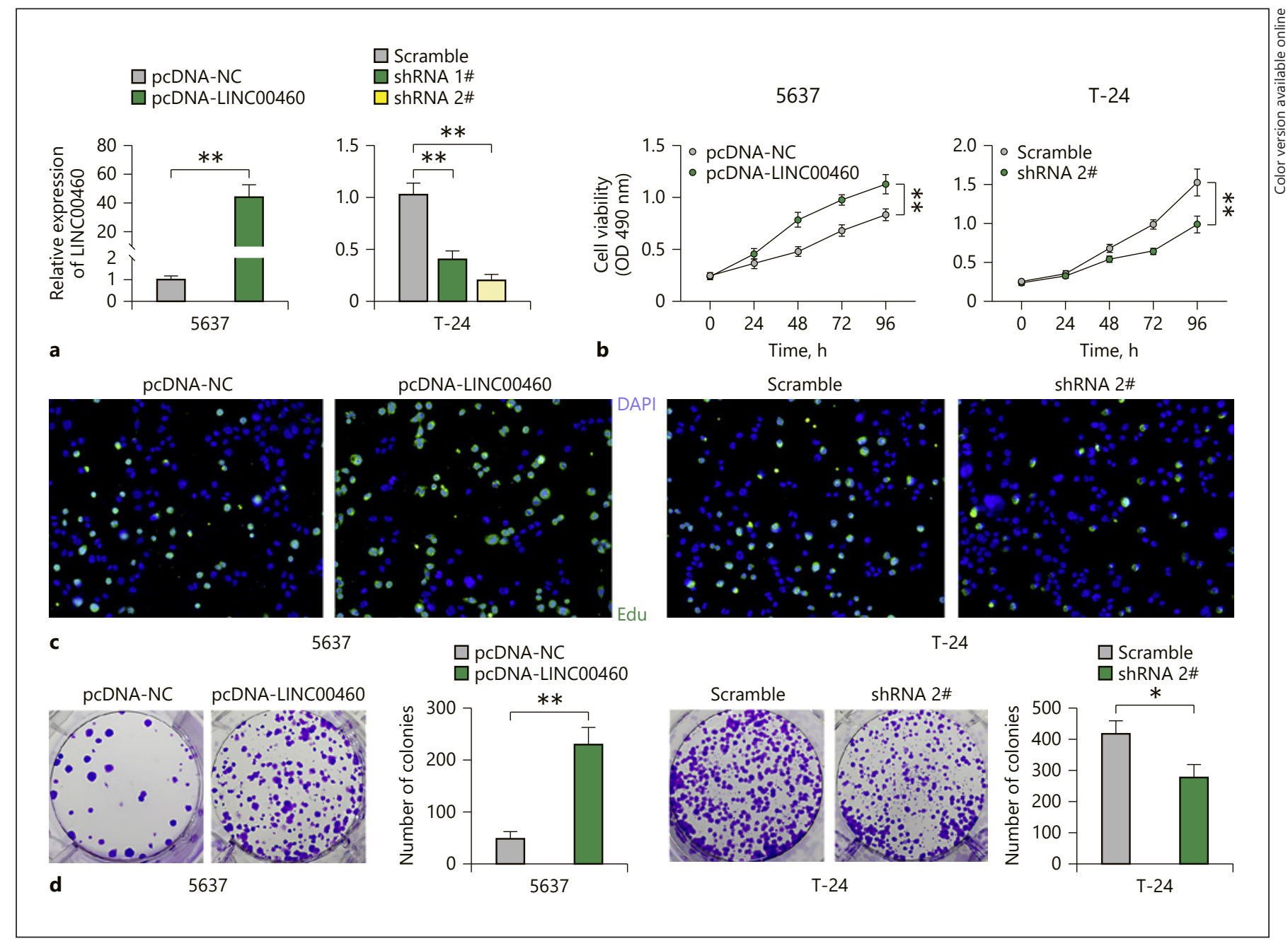

Fig. 2. LINC00460 promoted cell proliferation of bladder carcinoma. a Transfection efficiency of pcDNA-LINC00460 in 5637 cells or shRNA 1\# and 2\# in T-24 cells was detected by qRT-PCR. ** represents sh-RNAs versus Scramble or pcDNA-LINC00460 versus pcDNA-NC, $p<0.01$. b LINC00460 promoted cell viability of 5637 and T-24 cells, while knockdown of LINC00460 decreased cell viability, as detected by MTT. ** represents sh-RNAs versus Scramble or pcDNA-LINC00460 versus pcDNA-NC, $p<0.01$.

\section{Mouse Xenograft Assay}

The research was conducted in accordance with the guidelines of Guide for the Care and Use of Laboratory Animals of the National Institutes of Health and approved by the Medical Ethics Committee of The Second Affiliated Hospital of Fujian Medical University. Ten 4-week-old female BALB/c nude mice (20-25 g) were randomly divided into 2 groups. $100-\mu \mathrm{L} 5 \times 10^{6} \mathrm{~T}-24$ cells transfected with shLINC00460 $2 \#$ or shNC were subcutaneously injected in the right flank of the nude mice. Tumors were measured every 3 days. Tumor volume was calculated. Twenty days later, the mice were sacrificed and the tumor tissues were isolated and weighted. RNAs and proteins were extracted for analysis. c LINC00460 promoted cell proliferation of 5637 and T-24 cells, while knockdown of LINC00460 inhibited cell proliferation, as detected by the EdU staining assay. d LINC00460 promoted cell proliferation of 5637 and T-24 cells, while knockdown of LINC00460 inhibited cell proliferation, as detected by the colony formation assay. ${ }^{*}, * *$ represent sh-RNAs versus Scramble or pcDNALINC00460 versus pcDNA-NC, $p<0.05, p<0.01$.

\section{Immunohistochemistry}

Fixed and paraffined tumor tissues from the mice were first dewaxed and rehydrated and then blocked in $3 \% \mathrm{H}_{2} \mathrm{O}_{2}$. After immersed in Tris-EDTA buffer containing $0.05 \%$ Tween 20 (pH 9.0), the tumor sections were incubated in $4 \%$ dry milk and $0.3 \%$ goat serum and then incubated overnight with anti-FOXK1, anti-Ki67, and anti-E-cadherin antibody (Abcam). After incubation with HRP goat anti-rabbit IgG secondary antibody, the slides were examined under a light microscope (Olympus) with hematoxylin to stain cell nuclei. 


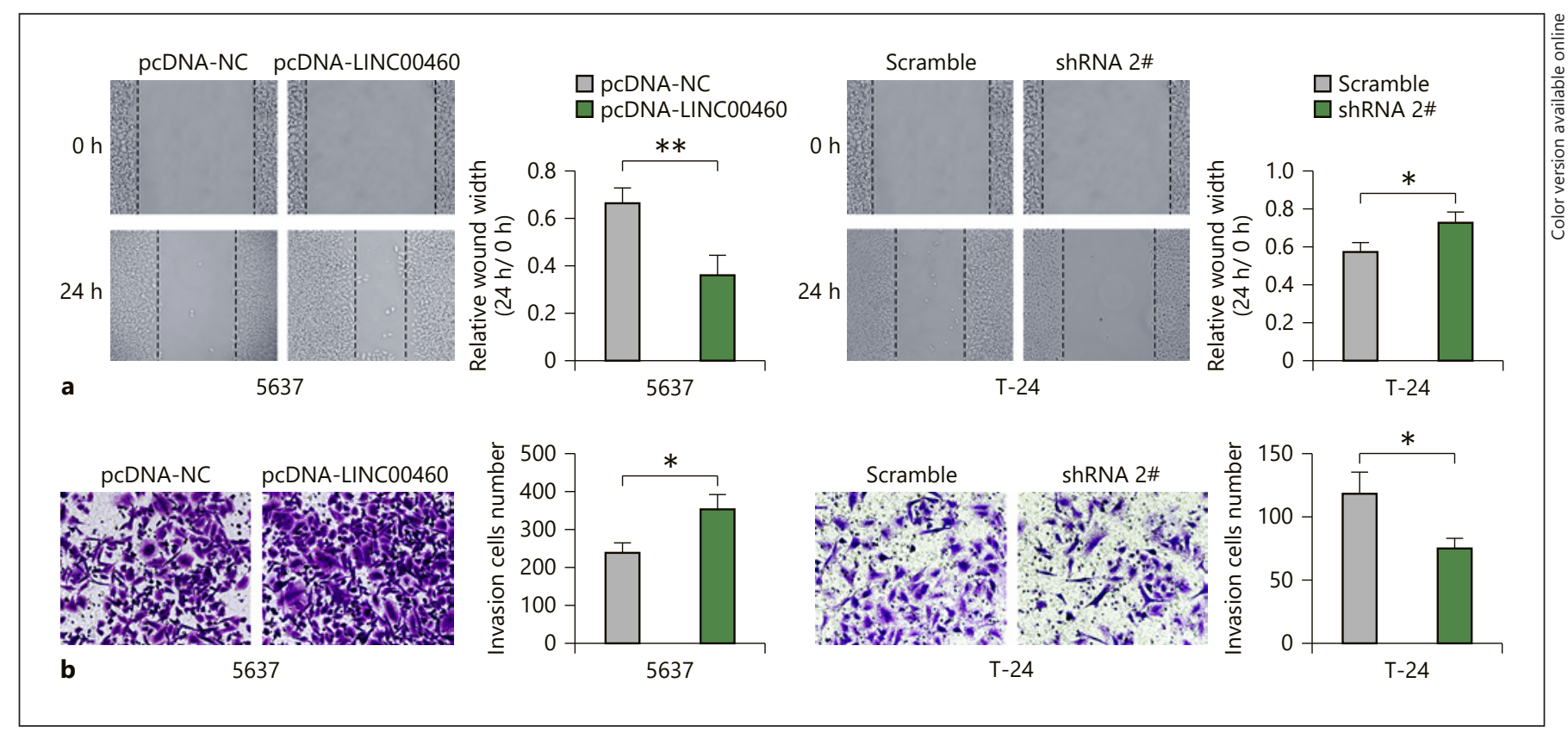

Fig. 3. LINC00460 promoted cell migration and invasion of bladder carcinoma. a LINC00460 promoted cell migration of 5637 and T-24 cells, while knockdown of LINC00460 inhibited cell migration, as detected by the wound healing assay. ${ }^{*},{ }^{*}$ represent shRNAs versus Scramble or pcDNA-LINC00460 versus pcDNA-

NC, $p<0.05, p<0.01$. b LINC00460 promoted cell invasion of 5637 and T-24 cells, while knockdown of LINC00460 inhibited cell invasion as detected by the transwell assay. ${ }^{*}$ represents sh-RNAs versus Scramble or pcDNA-LINC00460 versus pcDNA-NC, $p<$ 0.01 .

Statistical Analysis

Results are expressed as mean \pm SEM, and the statistical analyses were determined via GraphPad Prism software and 1-way analysis of variance. $p<0.05$ was considered as a mark of statistically significant.

\section{Results}

\section{LINC00460 Was Elevated in Bladder Carcinoma}

Tissues and Cell Lines

Dysregulation of LINC00460 was observed in bladder carcinoma tissues compared to adjacent noncancer tissues (Fig. 1a). Highly expressed LINC00460 indicated a significantly shorter overall survival (OS) of patients with bladder carcinoma than that of patients with lowly expressed LINC00460 ( $p=0.0473$ ) (Fig. 1b). Moreover, highly expressed LINC00460 was significantly related to tumor range $(p=0.026)$, metastasis $(p=0.019)$, lymph nodes $(p=0.002)$, and pathological stage $(p=0.016)$ (Table 1), suggesting that LINC00460 might contribute to metastasis or poor prognosis of bladder carcinoma. Meanwhile, LINC00460 was also elevated in bladder carcinoma cell lines (T-24, 5637, SW780, and RT-112) compared to SV-HIUC-1 (Fig. 1c). T-24 cells with highest
LINC00460 expression was selected for the subsequent loss-of-function assays, while 5637 with lowest expression was selected for the gain-of-function assays.

\section{LINC00460 Promoted Cell Proliferation, Migration, and Invasion of Bladder Carcinoma}

The effect of LINC00460 on cell proliferation of bladder carcinoma was investigated. First, 5637 cells were transfected with pcDNA-LINC00460 and T-24 cells were transfected with shRNAs targeting LINC00460, and then the transfection efficacies were determined by qRT-PCR (Fig. 2a). shRNA 2\# with lower expression of LINC00460 was selected for further experiments. Second, the MTT assay (Fig. 2b) showed that overexpression of LINC00460 increased cell viability of 5637 cells, while knockdown of LINC00460 decreased cell viability of T-24 cells. Moreover, EdU labeling (Fig. 2c) and colony formation (Fig. 2d) assays indicated that LINC00460 could promote cell proliferation of bladder carcinoma cells, while knockdown of LINC00460 suppressed cell proliferation. Finally, wound healing (Fig. 3a) and transwell (Fig. 3b) assays indicated that LINC00460 promoted cell migration and invasion of 5637 cells, while knockdown of LINC00460 inhibited cell migration and invasion of T-24 cells. In general, 
Table 2. Relationship between LINC00460 and clinicopathological parameters

\begin{tabular}{|c|c|c|c|c|}
\hline \multirow[t]{2}{*}{ Parameters } & \multirow{2}{*}{$\begin{array}{l}\text { Patients, } \\
n\end{array}$} & \multicolumn{2}{|c|}{ LINC00460 expression } & \multirow[t]{2}{*}{$p$ value } \\
\hline & & $\begin{array}{l}\text { low } \\
(<\text { median })\end{array}$ & $\begin{array}{l}\text { high } \\
\text { ( } \geq \text { median) }\end{array}$ & \\
\hline $\mathrm{N}$ & 43 & 21 & 22 & \\
\hline \multicolumn{5}{|l|}{ Age, years } \\
\hline$<60$ & 18 & 8 & 10 & \multirow[t]{2}{*}{0.625} \\
\hline$\geq 60$ & 25 & 13 & 12 & \\
\hline \multicolumn{5}{|l|}{ Gender } \\
\hline Male & 23 & 12 & 11 & \multirow[t]{2}{*}{0.639} \\
\hline Female & 20 & 9 & 11 & \\
\hline \multicolumn{5}{|l|}{ Smoking } \\
\hline No smoking & 15 & 6 & 9 & \multirow[t]{2}{*}{0.396} \\
\hline Smoking & 28 & 15 & 13 & \\
\hline \multicolumn{5}{|l|}{ Tumor range } \\
\hline $\mathrm{T} 1-\mathrm{T} 3$ & 30 & 18 & 12 & \multirow[t]{2}{*}{$0.026^{*}$} \\
\hline $\mathrm{T} 4$ & 13 & 3 & 10 & \\
\hline \multicolumn{5}{|l|}{ Metastasis } \\
\hline Negative & 25 & 16 & 9 & \multirow{2}{*}{$0.019^{*}$} \\
\hline Positive & 18 & 5 & 13 & \\
\hline \multicolumn{5}{|l|}{ Lymph nodes } \\
\hline Negative & 27 & 18 & 9 & \multirow[t]{2}{*}{$0.002^{*}$} \\
\hline Positive & 16 & 3 & 13 & \\
\hline \multicolumn{5}{|c|}{ Pathological stage } \\
\hline$<\mathrm{IV}$ & 27 & 17 & 10 & \multirow[t]{2}{*}{$0.016^{*}$} \\
\hline IV & 16 & 4 & 12 & \\
\hline
\end{tabular}

$* p<0.05$.

LINC00460 may account for malignant phenotypes of bladder carcinoma (Table 2).

\section{Negative Correlation between LINC00460 and}

miR-612

The potential binding target of LINC00460 was predicted as miR-612 (Fig. 4a). To determine the binding ability between LINC00460 and miR-612, 5637 cells transfected with miR-612 inhibitor and T-24 cells transfected with miR-612 mimics were validated by qRT-PCR (Fig. 4b). The dual luciferase reporter assay demonstrated that miR-612 mimics decreased luciferase activity of pmirGLO-wt-LINC00460, while miR-612 inhibitor increased luciferase activity (Fig. 4c). However, both miR612 mimics and inhibitor had no significant effects on luciferase activity of pmirGLO-mut-LINC00460 (Fig. 4c). Moreover, the expression of miR-612 was reduced in 5637 cells transfected with pcDNA-LINC00460, while increased in T-24 cells transfected with LINC00460 shRNA (Fig. 4d), suggesting that LINC00460 could directly bind with miR-612 and inhibit its expression. MiR-612 was found to be downregulated in bladder carcinoma tissues (Fig. 4e) and showed a negative correlation with LINC00460 (Fig. 4f).

\section{FOXK1 Was a Direct Target of miR-612}

FOXK1 was predicted as a miR-612-binding target (Fig. 5a). MiR-612 mimics decreased luciferase activity of pmirGLO-wt-FOXK1, while miR-612 inhibitor increased luciferase activity (Fig. 5b). However, both miR-612 mimics and inhibitor had no significant effects on luciferase activity of pmirGLO-mut-FOXK1 (Fig. 5b). Upregulation of FOXK1 in patients with bladder carcinoma was observed (Fig. 5c), which revealed a positive correlation with LINC00460 and a negative correlation with miR-612 (Fig. 5d).

\section{LINC00460 Promoted Bladder Carcinoma Progression} via Sponging miR-612-Mediated FOXK1

The effect of LINC00460/miR-612 axis on FOXK1 was first evaluated. Overexpression of LINC00460 increased the protein expression of FOXK1, while knockdown of LINC00460 decreased FOXK1 (Fig. 6a). Moreover, miR612 reversed the effect of LINC00460 on FOXK1 expression (Fig. 6a). To identify whether FOXK1 was necessary for LINC00460-mediated bladder carcinoma progression, T-24 cells were cotransfected with shRNA targeting LINC00460 and pcDNA-FOXK1. The results indicated that overexpression of FOXK1 increased shLINC00460inhibited cell viability of T-24 cells (Fig. 6b). In addition, the inhibitory ability of shLINC00460 on cell proliferation of T-24 cells was also reversed by overexpression of FOXK1 (Fig. 6c, d). Moreover, overexpression of FOXK1 counteracted the suppressive effect of LINC00460 silencing on migration (Fig. 6e) and invasion (Fig. 6f) of T-24 cells. Overexpression of FOXK1 attenuated the interference of LINC00460-induced PCNA decrease and E-cadherin increase in T-24 cells (Fig. 6g).

\section{Silencing of LINC00460 Inhibited in vivo Bladder \\ Carcinoma Tumor Growth}

T-24 cells transfected with shLINC00460 2\# were inoculated into the nude mice to investigate the in vivo functional role of LINC00460 in bladder carcinoma. Tumor growth was suppressed in the mice intratumorally injected with shLINC00460 2\# (Fig. 7a), as demonstrated by a decrease in tumor volume and weight (Fig. 7a). Moreover, xenograft tumor tissues injected with shLINC00460 2\# reduced the expression of FOXK1 and Ki67, while increased E-cadherin (Fig. 7b), suggesting 


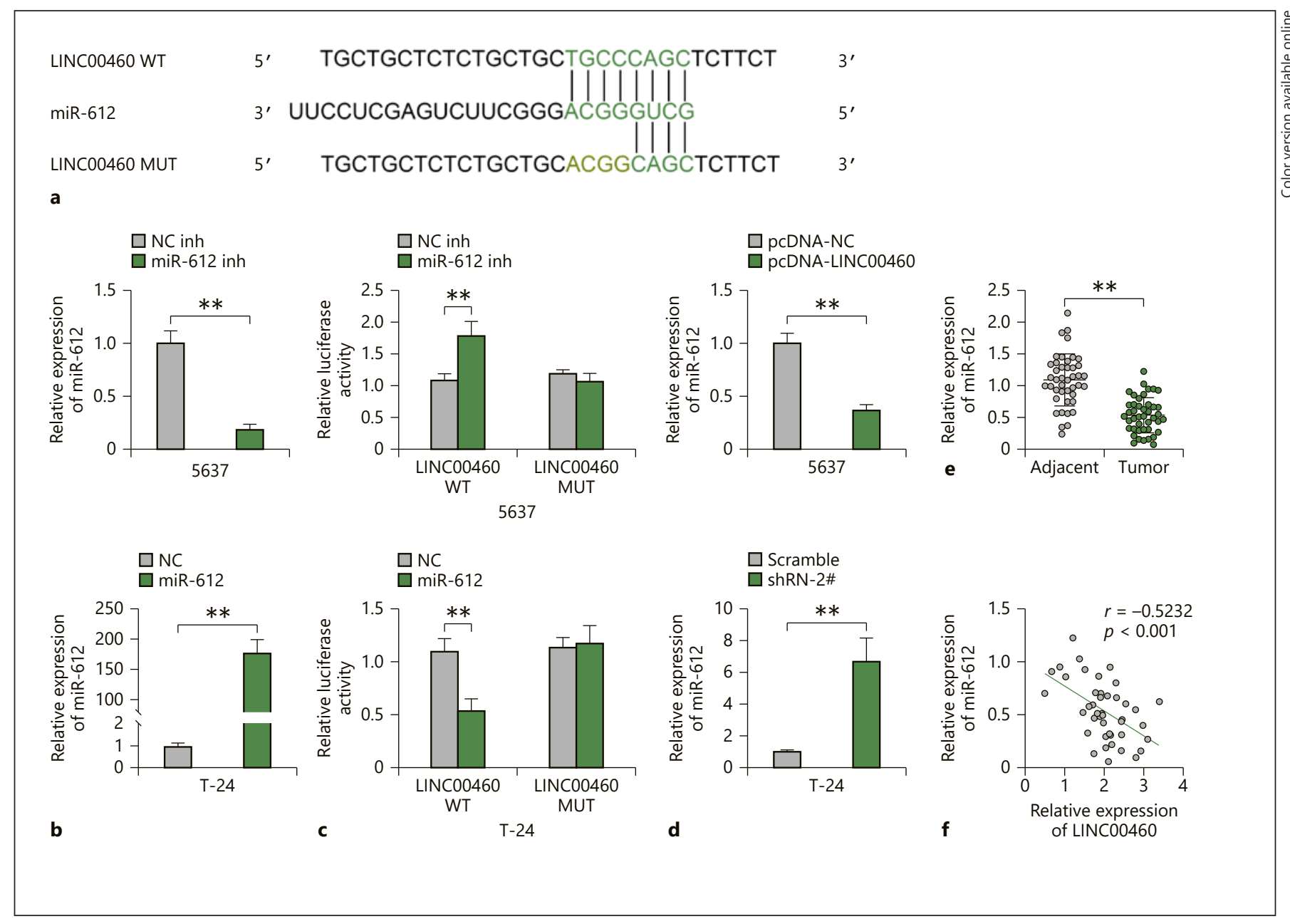

Fig. 4. Negative correlation between LINC00460 and miR-612. a The potential binding target of LINC00460 was predicted as miR-612 by starbase (http://starbase.sysu.edu.cn/index.php). b Transfection efficiency of miR-612 inhibitor in 5637 cells or miR-612 mimics in T-24 cells was detected by qRT-PCR. ** represents miR-612 inh versus NC inh or miR-612 versus NC, $p<0.01$. c MiR-612 mimics increased luciferase activity of pmirGLO-wtLINC00460, while miR-612 inhibitor decreased luciferase activities in 5637 or T-24 cells. ${ }^{* *}$ represents miR-612 inh versus NC inh

that silencing of LINC00460 inhibited in vivo bladder carcinoma tumor growth via downregulation of FOXK1.

\section{Discussion}

Dysregulated expression of lincRNAs has been reported in bladder carcinoma and employed to be biomarkers or therapeutic targets for bladder carcinoma [30]. Here, in line with previous study [15], the present study discovered an upregulated lincRNA, LINC00460, in bladder or miR-612 versus NC, $p<0.01$. $\mathbf{d}$ LINC00460 decreased miR-612 expression in 5637 or T-24 cells, while knockdown of LINC00460 increased miR-612. ${ }^{* *}$ represents sh-RNAs versus Scramble or pcDNA-LINC00460 versus pcDNA-NC, $p<0.01$. e MiR-612 was decreased in bladder carcinoma tissues compared to adjacent noncancer tissues, as detected by qRT-PCR. ${ }^{* *}$ represents Tumor versus Adjacent tissues, $p<0.01$. $\mathbf{A}$ A negative correlation between miR-612 and LINC00460 in bladder carcinoma patients was observed.

carcinoma tissues. Consistent with Wen et al. [16], the expression of LINC00460 was positively related to tumor range, metastasis, and lymph nodes of bladder carcinoma and predicted a poor prognosis in patients with bladder carcinoma. Then, the potential regulatory role of LINC00460 in bladder carcinoma progression was evaluated to clarify a potential therapeutic target in treatment of bladder carcinoma.

In addition to being a potential biomarker for the diagnosis of bladder carcinoma, LINC00460 also promoted cell proliferation and migration of bladder carcinoma 
[16]. Data from this study also showed that LINC00460 promoted cell proliferation, migration, and invasion of bladder carcinoma. Moreover, interference of LINC00460 suppressed bladder carcinoma progression. An in vivo subcutaneous xenotransplanted tumor model confirmed the inhibitory role of LINC00460 silencing in in vivo tumorigenic ability of bladder carcinoma. These results suggested the potential clinical application of LINC00460 on bladder carcinoma. However, the influence of LINC00460 on cell apoptosis of bladder carcinoma needs to be further investigated.

LncRNAs function as competing endogenous RNAs (CeRNAs) via binding with miRNAs to regulate the expression of target genes during bladder development
Fig. 5. FOXK1 was a direct target of miR612. a The potential miR-612 binding target was predicted as FOXK1 by Targetscan (http://www.targetscan.org/vert_71/). b MiR-612 mimics increased luciferase activity of pmirGLO-wt-FOXK1, while miR612 decreased the luciferase activity in 5637 or T-24 cells. ** represents miR-612 inh versus NC inh or miR-612 versus NC, $p<$ 0.01. c The mRNA expression of FOXK1 was increased in bladder carcinoma tissues and adjacent noncancer tissues, as detected by qRT-PCR. ** represents tumor versus adjacent tissues, $p<0.01$. d A positive correlation between LINC00460 and FOXK1 or a negative correlation between miR-612 and FOXK1 in bladder carcinoma patients was observed.

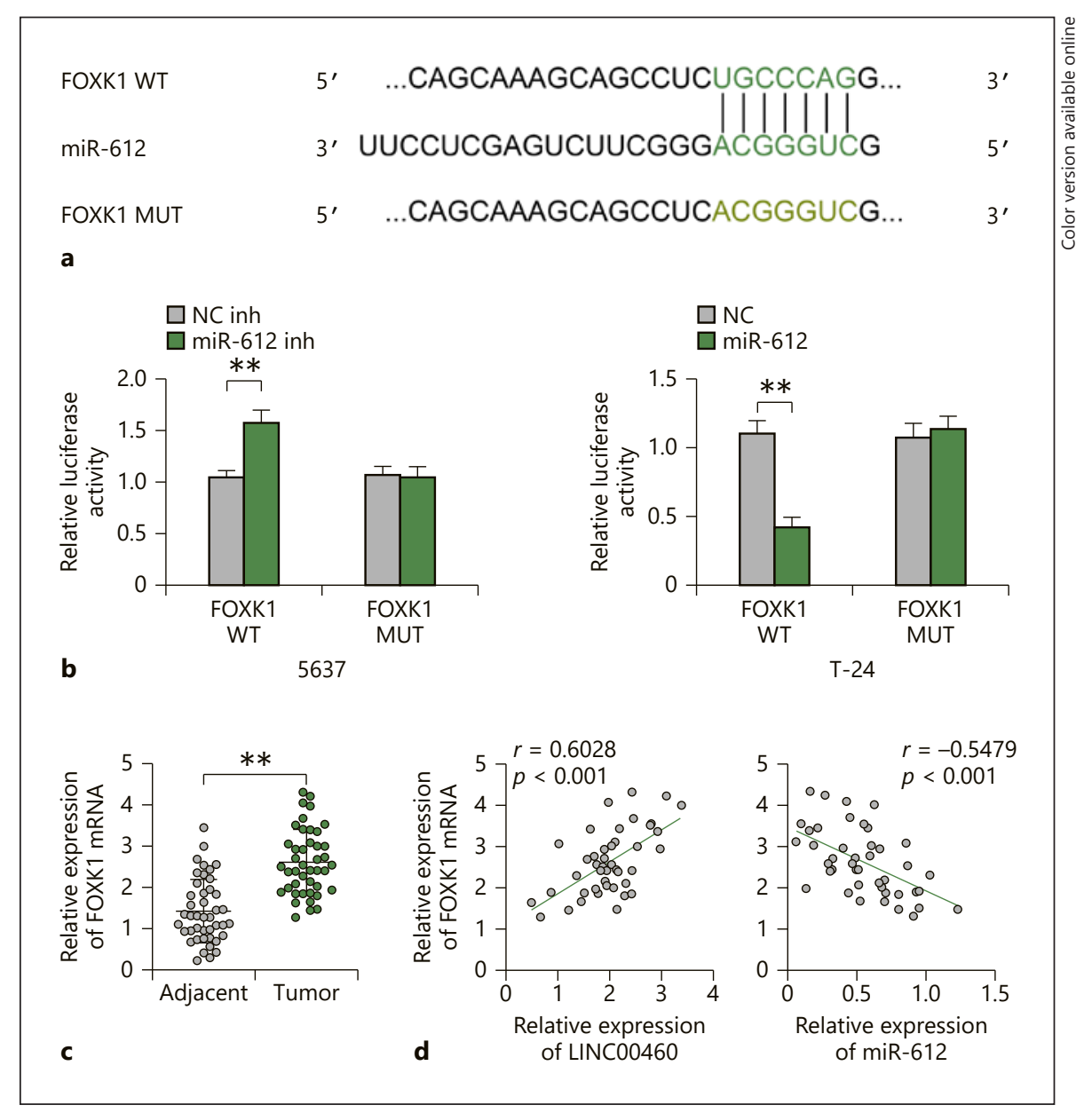

Fig. 6. LINC00460 promoted bladder carcinoma progression via sponging miR-612-mediated FOXK1. a The influence of LINC00460 and miR-612 on protein expression of FOXK1 in 5637 and T-24 cells was detected. ${ }^{* *}$ represents pcDNA-LINC00460+ miR-612 or pcDNA + NC versus pcDNA-LINC00460 + NC, $p<$ 0.01 . ${ }^{* *}$ represents sh-RNA $2 \#+$ miR-612 inh or Scramble $+\mathrm{NC}$ inh versus sh-RNA $2 \#+\mathrm{NC}$ inh, $p<0.01$. b The influence of LINC 00460 and FOXK1 on cell viability of 5637 and T-24 cells was detected by MTT. ** represents sh-RNA $2 \#+$ miR-612 inh or Scramble + NC inh versus sh-RNA $2 \#+$ NC inh, $p<0.01$. c The influence of LINC00460 and FOXK1 on cell proliferation of 5637 and T-24 cells was detected by EdU staining. d The influence of LINC00460 and FOXK1 on cell proliferation of 5637 and T-24 cells was detected by colony formation. ${ }^{* *}$ represents sh-RNA $2 \#+$ miR612 inh or Scramble + NC inh versus sh-RNA 2\# + NC inh, $p<0.01$. e The influence of LINC00460 and FOXK1 on cell migration of 5637 and T-24 cells was detected by wound healing. * represents sh-RNA $2 \#+$ miR-612 inh or Scramble + NC inh versus sh-RNA $2 \#+$ NC inh, $p<0.05$. $\mathbf{f}$ The influence of LINC00460 and FOXK1 on cell invasion of 5637 and T-24 cells was detected by transwell. * represents sh-RNA 2\# + miR-612 inh or Scramble + NC inh versus sh-RNA $2 \#+$ NC inh, $p<0.05$. g The influence of LINC00460 and FOXK1 on protein expression of FOXK1, PCNA, and E-cadherin in 5637 and T-24 cells was examined. ${ }^{* *}$ represents sh-RNA $2 \#+$ miR-612 inh or Scramble + NC inh versus sh-RNA 2\# + NC inh, $p<0.01$.
(For figure see next page.)
86

Pharmacology 2021;106:79-90 DOI: $10.1159 / 000509255$
Li/Huang/Zhang/Zhuo/Tong/Cai 


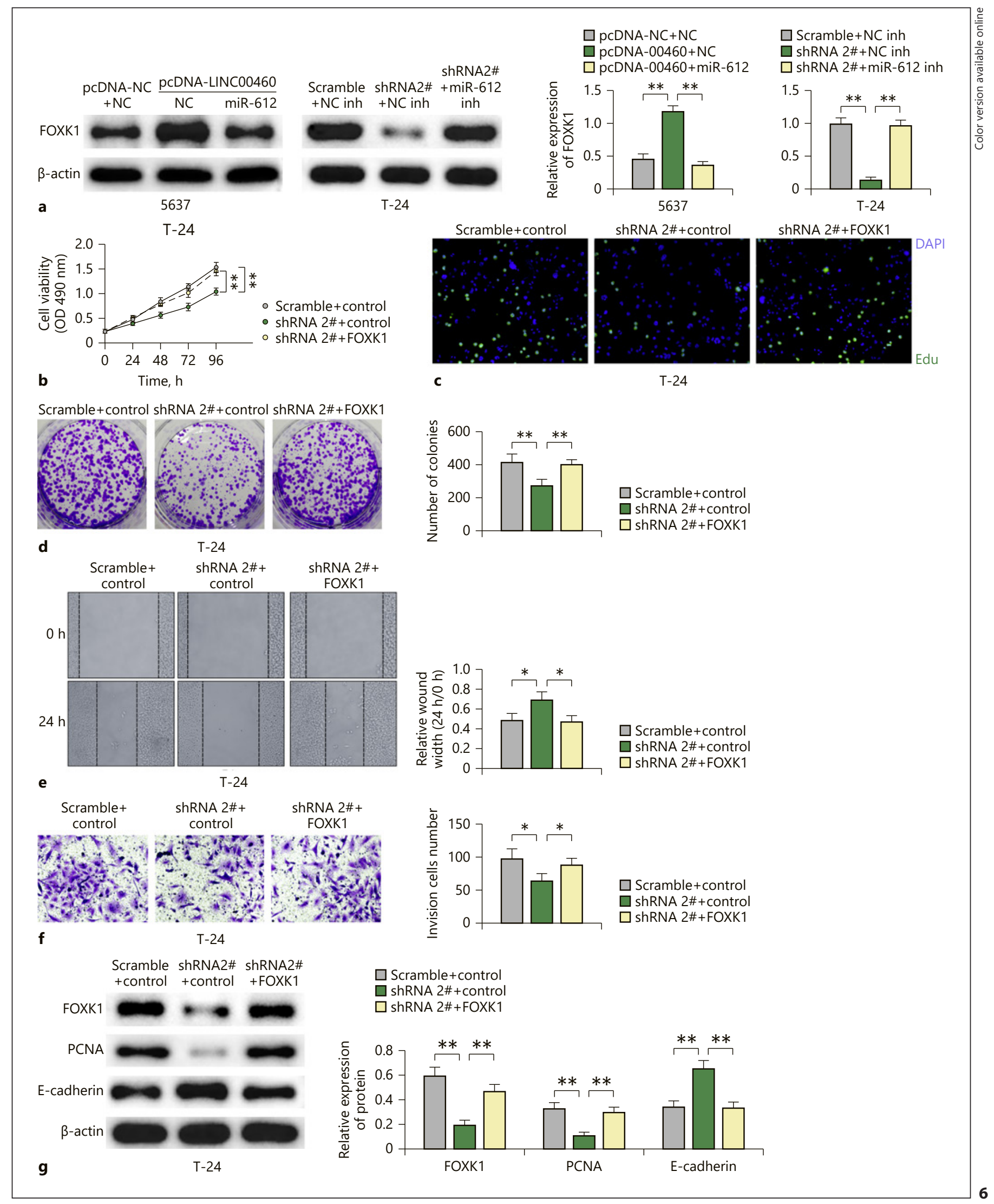

LINC00460/miR-612/FOXK1 Axis in

Pharmacology 2021;106:79-90 DOI: $10.1159 / 000509255$ 


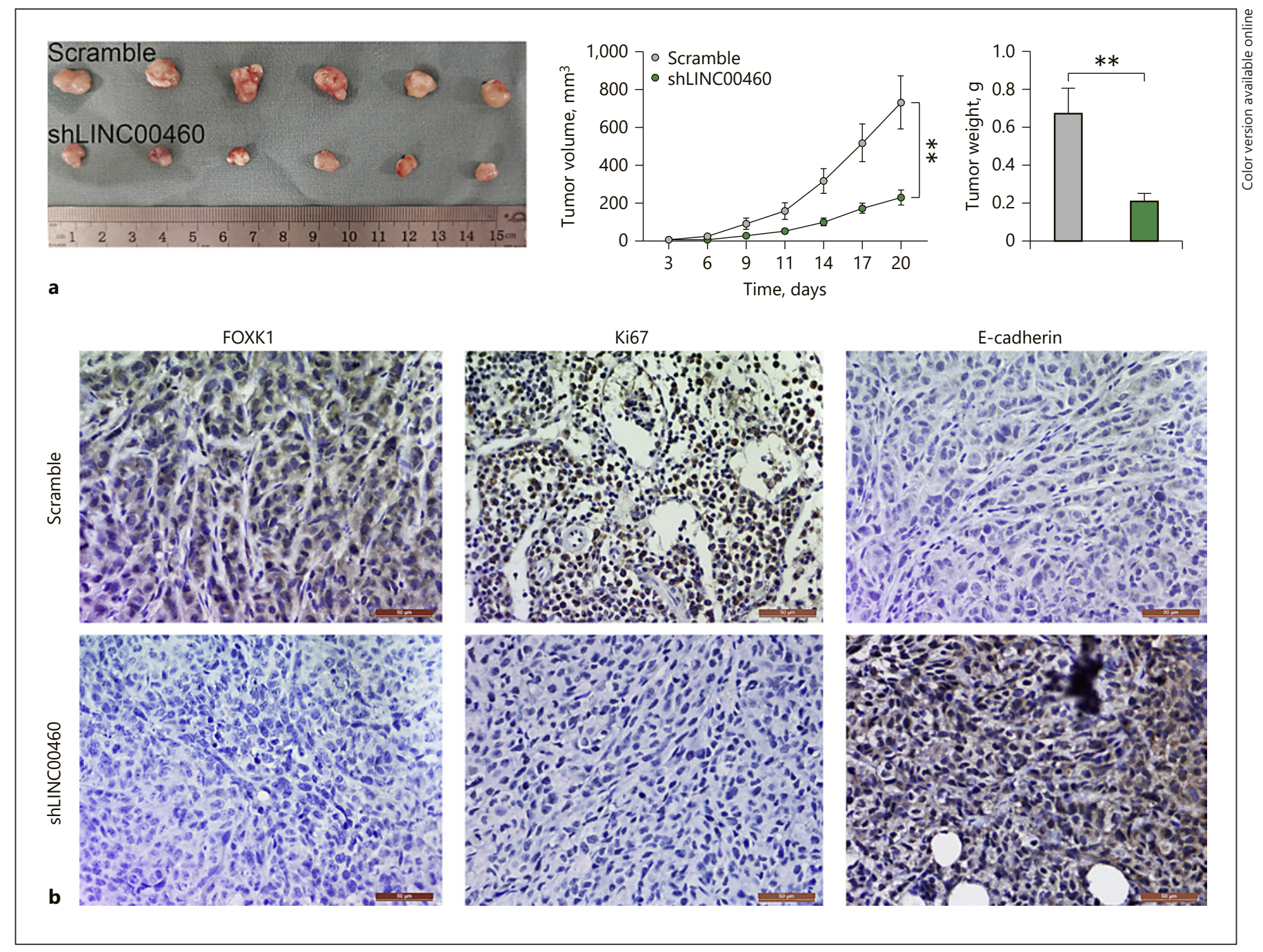

Fig. 7. Silencing of LINC00460 inhibited in vivo bladder carcinoma tumor growth. a Sh-LINC00460 suppressed bladder carcinoma tumor growth with decreased tumor volume and weight in xenograft tumor mice. ${ }^{* *}$ represents sh-LINC00460 versus Scram- ble, $p<0.01$. b Immunohistochemistry staining was used to determine expression of FOXK1, Ki-67, and E-cadherin affected by shLINC00460. Scale bar, $200 \mu \mathrm{m}$.
[31]. MiR-503, miR-143, miR-429, and miR-489 were reported to be involved in the CeRNA network of LINC00460 [15]. LINC00460 could sponge miR-612 to promote progression of head and neck squamous [32]. Here, miR-612 was validated as the target of LINC00460, and LINC00460 inhibited miR-612 expression. Moreover, miR-612 showed a negative correlation with LINC00460 in bladder carcinoma tissues. MiR-612 was tumor suppressor in liver cancer [33] and non-small-cell lung cancer [34]. Through targeting malic enzyme 1, miR-612 inhibited cell proliferation, migration, invasion, and epithelial-mesenchymal transition of bladder carci- noma [35]. EMT could be essential for metastasis in bladder carcinoma [36] by decrease of the epithelial marker, E-cadherin [37]. Although our results indicated that knockdown of LINC00460 decreased E-cadherin both in in vitro and in vivo experiments, the effect of LINC00460 on mesenchymal markers, including $\mathrm{N}$-cadherin and vimentin, should also be further investigated.

Furthermore, FOXK1 was found to be a new binding target of miR-612 in bladder carcinoma, and miR-612 inhibited the expression of FOXK1. Although the regulation of FOXK1 on bladder carcinoma has not been reported, FOXK1 could function as "oncogene" in colorec-
88

Pharmacology 2021;106:79-90 DOI: $10.1159 / 000509255$
Li/Huang/Zhang/Zhuo/Tong/Cai 
tal cancer [38], esophageal cancer [39], liver cancer [40], and prostate cancer [41]. In vitro functional assays showed that overexpression of FOXK1 counteracted the suppressive effect of LINC00460 silencing on bladder carcinoma progression. In vivo tumor model also indicated that silencing of LINC00460 inhibited in vivo bladder carcinoma tumor growth via downregulation of FOXK1. However, FOXK1 was also a tumor suppressor in breast cancer [42], and the downstream target gene of FOXK1 involved in LINC00460-mediated bladder carcinoma progression needs further investigation.

In conclusion, this study demonstrated that knockdown of LINC00460 inhibited cell proliferation, migration, and invasion of bladder carcinoma via sponging miR-612-mediated FOXK1. These results illuminated a close correlation between LINC00460/miR-612/FOXK1 axis and bladder carcinoma progression, suggesting the potential application of LINC00460 in bladder carcinoma.

\section{Statement of Ethics}

All procedures performed in the studies involving human participants were approved by the Medical Ethics Committee of The Second Affiliated Hospital of Fujian Medical University. All ani- mal experiments were conducted in accordance with the guidelines of the Guide for the Care and Use of Laboratory Animals of the National Institutes of Health and approved by the Medical Ethics Committee of The Second Affiliated Hospital of Fujian Medical University.

\section{Conflict of Interest Statement}

The authors state that there are no conflicts of interest to disclose.

\section{Funding Sources}

This work was supported by Medical Innovation Project of Fujian Provincial Health Commission (Grant No. 2019-CXB-18).

\section{Author Contributions}

F.Z.C. and J.W.L. conceived and designed the experiments, S.H.H. and Y.M.Z. analyzed and interpreted the results of the experiments, and W.F.Z. and B.C.T. performed the experiments.

\section{References}

1 Nativ O, Dalal E, Sabo E, Aronson M, Hidas G, Nativ O. Halofuginone: a novel oral and intravesical agent for the treatment of nonmuscle invasive bladder cancer. J Mol Clin Med. 2018;1(4):213-7.

2 Antoni S, Ferlay J, Soerjomataram I, Znaor A, Jemal A, Bray F. Bladder cancer incidence and mortality: a global overview and recent trends. Eur Urol. 2017;71(1):96-108.

3 Xu SQ, Buraschi S, Morcavallo A, Genua M, Shirao T, Peiper SC, et al. A novel role for drebrin in regulating progranulin bioactivity in bladder cancer. Oncotarget. 2015;6(13): 10825-39.

4 Martin-Doyle W, Kwiatkowski DJ. Molecular biology of bladder cancer. Hematol Oncol Clin North Am. 2015;29(2):191-203.

5 Svoboda P. Long and small noncoding RNAs during oocyte-to-embryo transition in mammals. Biochem Soc Trans. 2017;45(5):111724.

6 Prensner JR, Chinnaiyan AM. The emergence of lncRNAs in cancer biology. Cancer Discov. 2011;1(5):391-407.

7 Luo H, Zhao X, Wan X, Huang S, Wu D. Gene microarray analysis of the lncRNA expression profile in human urothelial carcinoma of the bladder. Int J Clin Exp Med. 2014;7(5):124454.
8 Wang F, Li X, Xie X, Zhao L, Chen W. UCA1, a non-protein-coding RNA up-regulated in bladder carcinoma and embryo, influencing cell growth and promoting invasion. FEBS Lett. 2008;582(13):1919-27.

9 He W, Cai Q, Sun F, Zhong G, Wang P, Liu $\mathrm{H}$, et al. linc-UBC1 physically associates with polycomb repressive complex 2 (PRC2) and acts as a negative prognostic factor for lymph node metastasis and survival in bladder cancer. Biochim Biophys Acta. 2013;1832(10): 1528-37.

10 Ying L, Huang Y, Chen H, Wang Y, Xia L, Chen $Y$, et al. Downregulated MEG3 activates autophagy and increases cell proliferation in bladder cancer. Mol Biosyst. 2013;9(3):40711.

11 Liu Z, Wang W, Jiang J, Bao E, Xu D, Zeng Y, et al. Downregulation of GAS5 promotes bladder cancer cell proliferation, partly by regulating CDK6. PLoS One. 2013;8(9): e73991.

12 Liang Y, Wu Y, Chen X, Zhang S, Wang K, Guan X, et al. A novel long noncoding RNA linc00460 up-regulated by $\mathrm{CBP} / \mathrm{P} 300$ promotes carcinogenesis in esophageal squamous cell carcinoma. Biosci Rep. 2017;37(5): BSR20171019.
13 Li K, Sun D, Gou Q, Ke X, Gong Y, Zuo Y, et al. Long non-coding RNA linc00460 promotes epithelial-mesenchymal transition and cell migration in lung cancer cells. Cancer Lett. 2018;420:80-90.

14 Kong YG, Cui M, Chen SM, Xu Y, Xu Y, Tao ZZ. LncRNA-LINC00460 facilitates nasopharyngeal carcinoma tumorigenesis through sponging miR-149-5p to up-regulate IL6. Gene. 2018;639:77-84.

15 Xu Z, Wang C, Xiang X, Li J, Huang J. Characterization of mRNA expression and endogenous RNA profiles in bladder cancer based on The Cancer Genome Atlas (TCGA) database. Int Med J Exp Clin Res. 2019;25:304160.

16 Wen L, Zhang X, Bian J, Han L, Huang H, He $\mathrm{M}$, et al. The long non-coding RNA LINC00460 predicts the prognosis and promotes the proliferation and migration of cells in bladder urothelial carcinoma. Oncol Lett. 2019;17(4):3874-80.

17 Knoll M, Lodish HF, Sun L. Long non-coding RNAs as regulators of the endocrine system. Nat Rev Endocrinol. 2015;11(3):151-60.

$18 \mathrm{Li} \mathrm{G}$, Kong Q. LncRNA LINC00460 promotes the papillary thyroid cancer progression by regulating the LINC00460/miR-485-5p/Raf1 axis. Biol Res. 2019;52(1):61. 
19 Zhang Y, Liu X, Li Q, Zhang Y. IncRNA LINC00460 promoted colorectal cancer cells metastasis via miR-939-5p sponging. Cancer Manag Res. 2019;11:1779-89.

$20 \mathrm{Tu}$ J, Zhao Z, Xu M, Chen M, Weng Q, Ji J. LINC00460 promotes hepatocellular carcinoma development through sponging miR-485$5 \mathrm{p}$ to up-regulate PAK1. Biomed Pharmacother. 2019;118:109213.

21 Dai B, Gong A, Jing Z, Aldape KD, Kang SH, Sawaya R, et al. Forkhead box M1 is regulated by heat shock factor 1 and promotes glioma cells survival under heat shock stress. J Biol Chem. 2012;288(3):1634.

22 Stoll SW, Stuart PE, Swindell WR, Tsoi LC, Li B, Gandarillas A, et al. The EGF receptor ligand amphiregulin controls cell division via FoxM1. Oncogene. 2016;35(16):2075-86.

23 Kim TH, Jo SW, Lee YS, Kim YJ, Lee SC, Kim WJ, et al. Forkhead box O-class 1 and forkhead box G1 as prognostic markers for bladder cancer. J Korean Med Sci. 2009;24(3): $468-73$.

24 Xian S, Shang D, Kong G, Tian Y. FOXJ1 promotes bladder cancer cell growth and regulates Warburg effect. Biochem Biophys Res Commun. 2018;495(1):988-94.

25 Gao F, Tian J. FOXK1, regulated by miR-365$3 p$, promotes cell growth and EMT indicates unfavorable prognosis in breast cancer. Onco Targets Ther. 2020;13:623.

26 Long Z, Wang Y. miR-195-5p suppresses lung cancer cell proliferation, migration, and invasion via FOXK1. Technol Cancer Res Treat. 2020;19:153303382092258.

27 Zhang P, Tang WM, Zhang H, Li YQ, Peng Y, Wang J, et al. MiR-646 inhibited cell proliferation and EMT-induced metastasis by targeting FOXK1 in gastric cancer. Br J Cancer. 2017;117(4):525-34.
28 Ma X, Yang X, Bao W, Li S, Liang S, Sun Y, et al. Circular RNA circMAN2B2 facilitates lung cancer cell proliferation and invasion via miR-1275/FOXK1 axis. Biochem Biophys Res Commun. 2018;498(4):1009-15.

29 Lu SR, Li Q, Lu JL, Liu C, Xu X, Li JZ. Long non-coding RNA LINC01503 promotes colorectal cancer cell proliferation and invasion by regulating miR-4492/FOXK1 signaling. Exp Ther Med. 2018;16(6):4879-85.

30 Ousati Ashtiani Z, Pourmand G, Salami SA, Ayati M, Tavakkoly-Bazzaz J. Dysregulated expression of long intergenic non-coding RNAs (LincRNAs) in urothelial bladder carcinoma. Int J Mol Cell Med. 2017;6(4):21221.

31 Miao L, Liu HY, Zhou C, He X. LINC00612 enhances the proliferation and invasion ability of bladder cancer cells as ceRNA by sponging miR-590 to elevate expression of PHF14. J Exp Clin Cancer Res. 2019;38(1):143.

32 Xie X, Xiong G, Wang Q, Ge Y, Cui X. Long non-coding RNA LINC00460 promotes head and neck squamous cell carcinoma cell progression by sponging miR-612 to up-regulate AKT2. Am J Transl Res. 2019;11(10):6326.

33 Tang J, Tao ZH, Wen D, Wan JL, Liu DL, Zhang S, et al. MiR-612 suppresses the stemness of liver cancer via Wnt/ $\beta$-catenin signaling. Biochem Biophys Res Commun. 2014; 447(1):210-5

34 Kang X, Kong F, Wu S, Liu Q, Yang C, Wu X, et al. microRNA-612 suppresses the malignant development of non-small-cell lung cancer by directly targeting bromodomain-containing protein 4. Onco Targets Ther. 2019; 12:4167-79.
35 Liu M, Chen Y, Huang B, Mao S, Cai K, Wang $\mathrm{L}$, et al. Tumor-suppressing effects of microRNA-612 in bladder cancer cells by targeting malic enzyme 1 expression. Int J Oncol. 2018; 52(6):1923-33.

36 Franzen CA, Blackwell RH, Todorovic V, Greco KA, Foreman KE, Flanigan RC, et al. Urothelial cells undergo epithelial-to-mesenchymal transition after exposure to muscle invasive bladder cancer exosomes. Oncogenesis. 2015;4:e163.

37 Luo J, Chen J, Li H, Yang Y, Yun H, Yang S, et al. LncRNA UCA1 promotes the invasion and EMT of bladder cancer cells by regulating the miR-143/HMGB1 pathway. Oncol Lett. 2017;14(5):5556-62.

38 Wu Y, Xie R, Liu X, Wang J, Peng Y, Tang W, et al. Knockdown of FOXK1 alone or in combination with apoptosis-inducing 5 -FU inhibits cell growth in colorectal cancer. Oncol Rep. 2016;36(4):2151-9.

39 Chen D, Wang K, Li X, Jiang M, Ni L, Xu B, et al. FOXK1 plays an oncogenic role in the development of esophageal cancer. Biochem Biophys Res Commun. 2017;494(1-2):88-94.

40 Cui H, Gao Q, Zhang L, Han F, Wang L. Knockdown of FOXK1 suppresses liver cancer cell viability by inhibiting glycolysis. Life Sci. 2018;213:66-73.

41 Chen F, Xiong W, Dou K, Ran Q. Knockdown of FOXK1 suppresses proliferation, migration, and invasion in prostate cancer cells. Oncol Res. 2017;25(8):1261-7.

42 Sun T, Wang H, Li Q, Qian Z, Shen C. Forkhead box protein $\mathrm{k} 1$ recruits TET1 to act as a tumor suppressor and is associated with MRI detection. Jpn J Clin Oncol. 2016;46(3):20921. 